\title{
Remote-Engineering und bildgestützte Inbetriebnahme mit einer Web-SPS
}

\author{
M. Seitz, M. Khushairi, A. Jürgens und H. Peter ${ }^{1}$
}

\section{Zusammenfassung}

Schwer zugängliche Anlagen müssen per Fernwartung projektiert und in Betrieb genommen werden. Während der Corona-Krise rückten die hierfür zugrundeliegenden Technologien auch in den Blickpunkt der Lehre, um Studierenden, die keinen Zugang zu den Versuchsaufbauten an der Hochschule hatten, die Interaktion mit realen Anlagen zu ermöglichen.

Deshalb wurde an der Hochschule Mannheim ein System entwickelt, das Kommunikationskonzepte der Industrie 4.0 zur Vernetzung von Steuerungen im Internet, das cloudbasierte Engineering sowie die bildgestützte Inbetriebnahme an einer realen Experimentieranlage veranschaulicht.

Eine automatische Bildauswertung soll die Anlage überwachen, um Störfälle, die etwa durch Programmierfehler eintreten könnten, zu vermeiden. Aber auch schlecht eingestellte Regler oder unzureichend kalibrierte Sensorik, was z.B. durch Alterung verursacht sein kann, soll durch die Bildverarbeitung automatisch erkannt werden. Die Bildverarbeitung dient somit zur automatisierten Erfolgskontrolle der entwickelten Software und der in Betrieb genommen Anlage.

\section{Stichwörter}

Remote-Engineering, Web-SPS, Bildverarbeitung, Inbetriebnahme, Erfolgskontrolle

\section{Einleitung}

Die Vernetzung und Cloud-Anbindung von Automatisierungssystemen steht gegenwärtig im Fokus der Industrie 4.0. SPSen mit Edge-Interfaces ermöglichen eine Cloud-Anbindung und den Zugriff von Remote-PCs aus dem Internet.

Dabei ist die zugrundeliegende Technologie schon lange bekannt [1,2]. Fast alle Automatisierungssysteme bieten heutzutage eine Webvisualisierung an [3], auch das Remote-Engineering mit Zugriff auf die Steuerungshardware über das Internet mit entsprechenden Sicherheitsmechanismen ist Stand der Technik [4,5]. SPSen oder mini-PCs wie der Raspberry-Pi werden heute oft als Edge-Gateways verwendet, um Sensordaten an die Cloud zu senden und von dort Befehle zu erhalten [6,7].

Für eine Remote-Inbetriebnahme können Webcams zur Beobachtung der Anlage eingesetzt werden [4]. Aber die Integration von Bildverarbeitungssystemen in heutige SPSen steht noch am Anfang [8,9]. In diesem Beitrag wird deshalb ein System vorgestellt, das mit Hilfe von Bildverarbeitung eine automatisierte Remote-Inbetriebnahme ermöglicht. Der Anwender kann die Anlage aus der Ferne über eine Webcam beobachten und wird durch eine automatisierte Bildauswertung bei der Inbetriebnahme unterstützt.

Die Bildverarbeitung dient dabei zur Anlagenüberwachung und kann auch während des Betriebs Störungen infolge schlecht kalibrierter Sensoren oder schlecht eingestellter Regler erkennen. Anhand des Experimentalaufbaus nach Bild 1 werden Möglichkeiten und Grenzen des Einsatzes von Bildverarbeitung beim Remote-Engineering und bei der Remote-Inbetriebnahme untersucht.

\footnotetext{
${ }^{1}$ Hochschule Mannheim, Institut für industrielle Automatisierungssysteme
} 


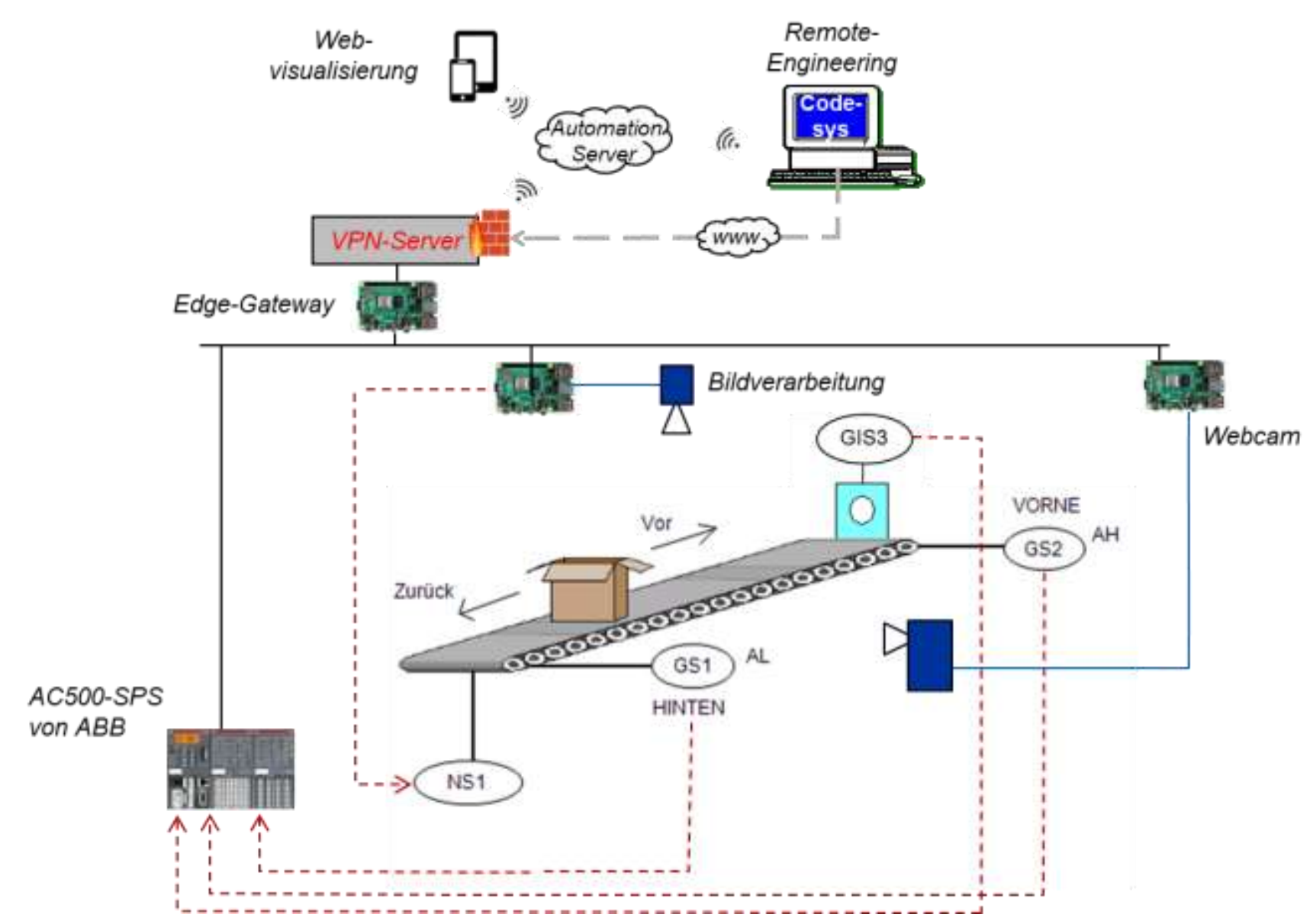

Bild 1: Aufbau des Experimentalsystems zum Remote Engineering. Das Förderband wird durch den Motor NS1 angetrieben, der wie die Endschalter GS1 und GS2 sowie der Ultraschallsensor GIS3 von einer SPS und einem Raspberry-Pi gesteuert werden

\section{Remote-Engineering für vernetzte Automatisierungssysteme}

Für ein Remote-Engineering gibt es verschiedene Möglichkeiten. Die meisten SPSen wie die AC500 in Bild 1 ermöglichen die Ankopplung eines Engineering-PCs über eine LAN- bzw. WLAN-Verbindung, so dass grundsätzlich von jedem PC im Steuerungsnetzwerk auf die SPS zugegriffen werden könnte. Natürlich muss das Netzwerk dann durch Firewall, Virenscanner, Authentifizierung und andere Maßnahmen der IT-Security nach IEC 62433 geschützt sein [10]. Durch einen VPN-Server können auch Rechner aus dem Internet in das virtuelle private Netzwerk integriert werden und auf die SPSen zugreifen.

\subsection{VPN-Server}

Für den Internet-basierten Zugriff auf die Steuerung läuft der Web-Server in der SPS. Mit Routern wird ein sog. VPN-Tunnel aufgebaut, der die Internet-Verbindung gegen Fremdeinwirkung schützt. Dabei wird jeweils ein Router im Segment des Servers und im Segment des Clients eingesetzt. Der VPN-Client kann auch als Software auf dem Engineering-PC laufen, wobei das Sicherheitsrisiko bei einer Softwarelösung immer etwas höher ist als bei der Realisierung mit Hardware. 
Die Router bieten Verschlüsselungssysteme für geschützte Verbindungen, wie das IPSec-Protokoll (Internet Protokoll Security). Mit dieser Verschlüsselung schafft man eine Verbindung, die von außen geschützt ist und intern die Möglichkeit gibt, alle Teilnehmer über lokale IP-Adresse anzusprechen [6]. Nach der Konfiguration des VPN/IPSec-Tunnels ist das Handling genau so, als wären die Teilnehmer am Engineering-PC durch ein Netzwerkkabel mit den Steuerungen verbunden.

\subsection{Web-SPS}

Das Förderband in Bild 1 kann von einer AC500-SPS oder einem Raspberry-Pi angesteuert werden. Auf beiden Systemen läuft Codesys, jedoch im ABB Automation Builder in einer älteren Version. In diesem Beitrag soll die Sensorik von der SPS eingelesen werden, ein Raspberry-Pi, der mit Hilfe einer Bildverarbeitung die Position der Kisten auf dem Förderband ermittelt, steuert den Förderbandantrieb. Beide können aus der Ferne über VPN programmiert werden.

Ein weiterer Raspberry-Pi liefert mit Hilfe einer Webcam Live-Bilder der Szene, so dass der Entwickler die Auswirkungen seiner Programmierung bei der Remote-Inbetriebnahme beobachten kann.

Die Raspberry-Pis können in den Codesys Automation Server eingebunden werden, die AC500 jedoch nicht. Um die Sensordaten nutzen zu können, sollen sie als Netzwerkvariablen von der AC500 an ein Codesys-Projekt im Raspberry-Pi gesendet werden, der in den Automation Server eingebunden ist und ein Cloud-basiertes Engineering ermöglicht.

In unserem Experimentalaufbau laufen die Raspberry-Pis aber in einer neueren Version als im ABB Automation Builder der SPS, so dass der Datenaustausch über UDP-Netzwerkvariablen zwischen der AC500 und dem Raspberry-Pi leider nicht funktionierte. Deshalb wurden im Verlauf der Experimente auch die Sensoren von dem Raspberry-Pi eingelesen, der auch die Bildverarbeitung ausführt.

Zum Bedienen und Beobachten der Anlage wird eine Codesys-HMI auf dem Raspberry-Pi realisiert, die auch eine Webvisualisierung ermöglicht. Somit kann der Anwender aus dem Web-Browser heraus ohne die Codesys-Entwicklungsumgebung die Anlage bedienen und beobachten (vgl. Bilder 3 und 6).

\subsection{Edge-Gateway und Cloud Engineering}

In großen Anlagen arbeiten viele Ingenieure an einem Projekt, auch in unserem Beispiel arbeiten viele Studierende mit der Förderbandanlage. Ein Planungsdokument wird oft von mehreren bearbeitet und verändert. Dazu ist es notwendig, die Dokumente in der Cloud abzulegen, in verschiedenen Versionen zu verwalten und die Zugriffsrechte zu regeln. Durch die zentrale Datenverwaltung hat der Benutzer den Vorteil, sich nicht um die Speicherung und Sicherheit seiner Daten selbst kümmern zu müssen, denn dies übernehmen die Services der Cloud.

Der Codesys Automation Server ermöglicht das Engineering in der Cloud. Dazu werden die SPS-Softwareprojekte über ein Edge-Gateway in der Cloud gespeichert. Der Entwickler sieht wie in Bild 2 dargestellt die eingesetzten SPSen und deren Betriebszustand. Außerdem sieht er die auf den SPSen laufenden Applikationen, die er von seinem Remote-PC aus mit dem Programmiersystem öffnen, verändern und wieder in die SPS laden kann. Ein gemeinsamer Zugriff ist somit kollisionsfrei möglich, was beim einfachen Zugriff über VPN wie Abschnitt 2.1 und 2.2 beschrieben zu Problemen führen kann.

Durch ein Edge-Gateway stellt der Codesys Automation Server eine sichere Verbindung zwischen den SPSen und der Cloud her. Dabei werden die Daten verschlüsselt von den Steuerungen über ein Transport Layer Security (TLS-Protokoll) an die Cloud übertragen [4]. Dies ermöglicht wie in Bild 2 gezeigt die Verwaltung mehrerer Steuerungen und ihrer Software im Webbrowser auf PC, Tablet oder Smartphone. 


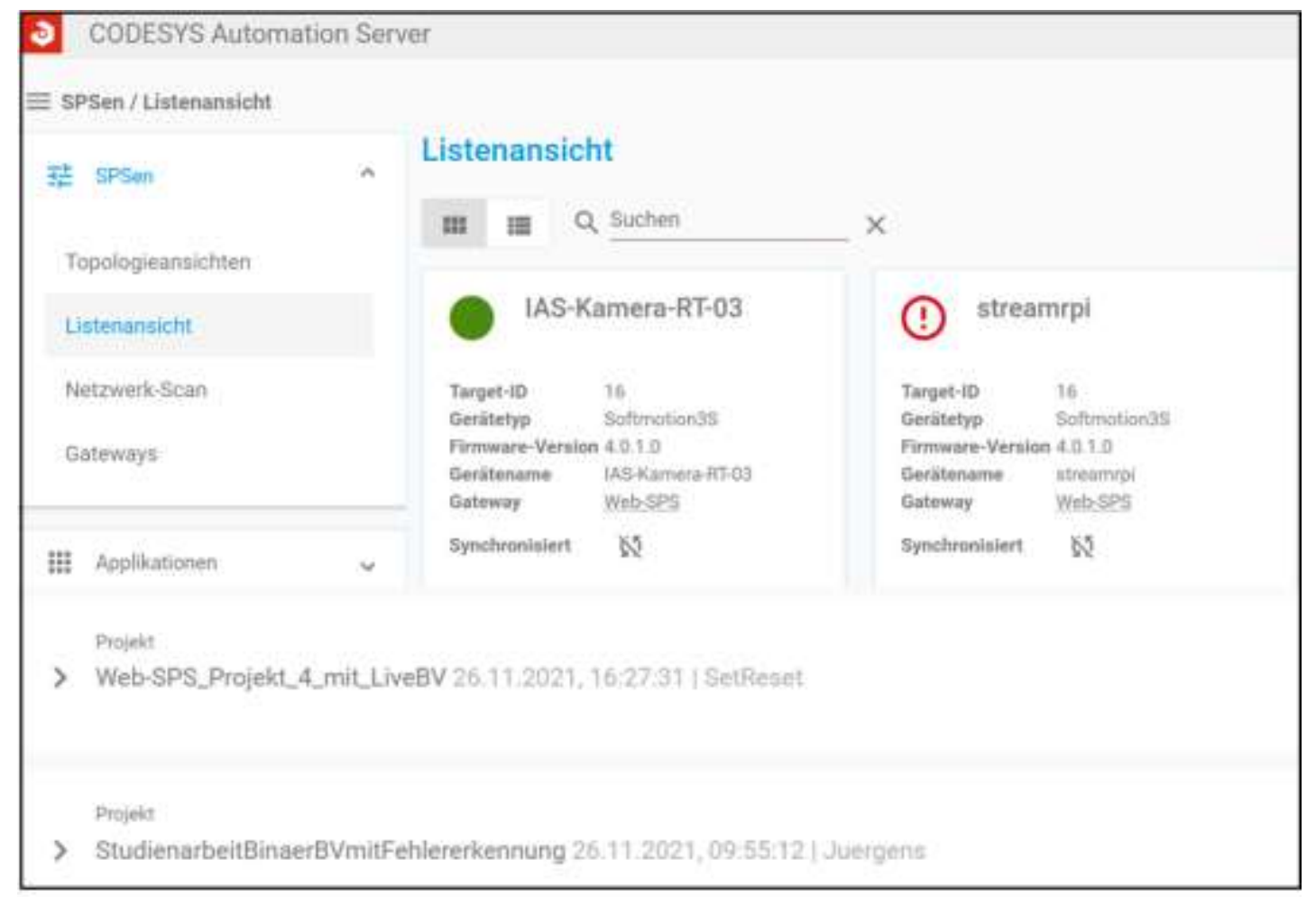

Bild 2: Cloud Engineering mit dem Codesys Automation Server, mit dem die Zustände der eingesetzten Hardwaremodule dargestellt und SPS-Projekte aus der Cloud geöffnet und bearbeitet werden können

\section{Bildgestützte Inbetriebnahme}

Eine Inbetriebnahme aus der Ferne benötigt Kameras, damit die Anwender die Bewegungen und Veränderungen in der Anlage beobachten können. Um nicht von der menschlichen Aufmerksamkeit abhängig zu sein, werden die Bilder automatisiert ausgewertet, so dass Fehler oder die erfolgreiche Ausführung der Automatisierung auch quantitativ bewertet werden.

\subsection{Streaming in die Prozessvisualisierung}

Handelsübliche Webcams liefern zwar Bilder als Live-Stream von der in der Ferne installierten Anlage, doch dazu ist meist eine mitgelieferte App erforderlich. Wünschenswert wäre die Einbindung des Live-Streams in die Prozessvisualisierung des Automatisierungssystems.

Mit einer am Raspberry-Pi angeschlossenen Webcam können Videostreams wie in Bild 3 veranschaulicht in der Webvisualisierung von Codesys dargestellt werden. Dazu wird der Videostream in eine $\mathrm{html}$-Datei eingebunden. In der Visualisierung wird ein Webbrowser-Element angelegt und der Name der html-Datei sowie die IP-Adresse des Raspberry als URL spezifiziert.

Wenn das Programm ausgeführt wird, kann das Webbrowser-Element in Bild 3 als Webclient die URL beim Webserver anfordern. Dieser überträgt dann die html-Datei an die Webvisualisierung, die dann die Webseite mit dem Videostream anzeigt.

Als Webcam wird eine Weitwinkelkamera eingesetzt, die zwar einen großen Bildbereich zeigt, aber auch starke Linsenverzerrungen (Fischaugeneffekt) verursacht. Wenn Anlagen über große Strecken verteilt oder schlecht zugänglich sind, ist es sinnvoll, mit einer zweiten genaueren Kamera, eine automatisierte Bildverarbeitung zur Anlagenüberwachung durchzuführen. 
a) Auschnitt der Webvisualisierung mit eingebettetem Videostream

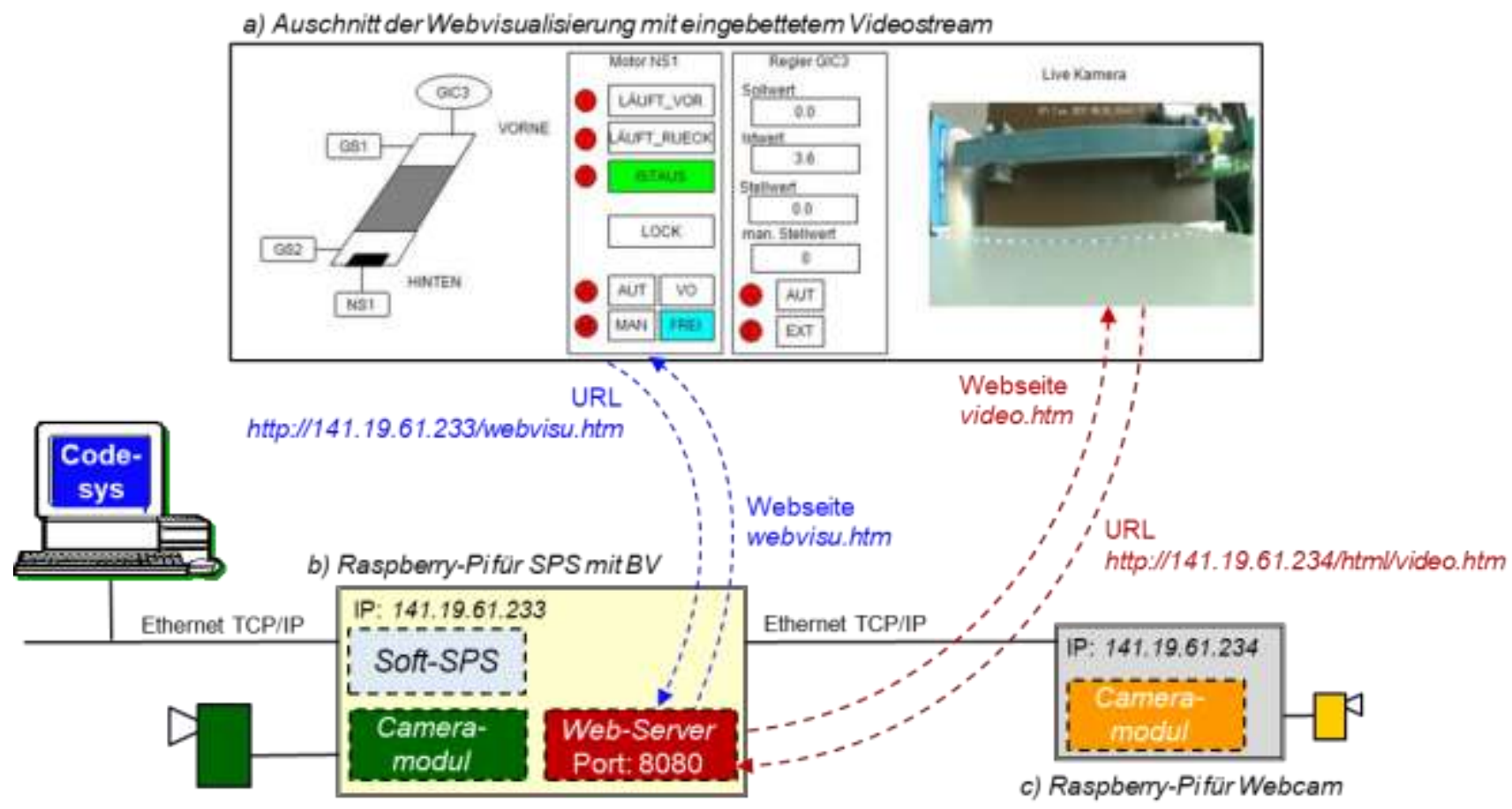

Bild 3: Anzeige- und Bedienoberfläche mit eingebettetem Video-Stream der Webcam

\subsection{Bildverarbeitung zur Erfolgskontrolle}

Zur Bildauswertung wird eine Kamera mit größerer Brennweite und entsprechend weniger Linsenverzerrungen eingesetzt. Diese kann z.B. analysieren, ob das transportierte Objekt die vorgegebene Sollposition erreicht hat oder ob der Antrieb an den Bandenden anhält, damit das Objekt nicht herunterfällt.

Da die zu überwachenden Positionen bekannt sind, wird die Bildverarbeitung wie in Bild 4 veranschaulicht quasi als Lichtschranke benutzt. Dabei wird ausgenutzt, dass sich auf dem dunklen Förderband vergleichsweise helle Objekte befinden. Dadurch kann in den relevanten Bildregionen die Anzahl heller Pixel gezählt werden, wodurch die Anwesenheit eines Objekts in einem Überwachungsbereich schnell erkannt wird.

Da in diesem Projekt eine Web-SPS eingesetzt wird und jeder Studierende seine Software aus der Ferne ausprobieren soll, kann kein reales Objekt auf dem Förderband transportiert werden, weil dies bei Programmierfehlern herunterfallen könnte und niemand vor Ort es wieder auf das Band setzen kann.

Deshalb wird ein flexibles Objekt aus Pappe auf das Band geklebt, so dass es auch über das Bandende hinaus und auf der Unterseite des Bandes wieder zurück auf die Oberseite fahren kann. Dieses Objekt berührt zwar nicht die Endschalter GS1 bzw. GS2 an den Bandenden, aber die Bildverarbeitung kann das erreichte Bandende erkennen und den Förderbandantrieb abschalten. Die Bildverarbeitung ersetzt somit die beiden Endschalter und kann auch den Ultraschall-Abstandssensor GIS3 ersetzen, indem sie an der vorgegebenen Sollposition die Anwesenheit des Objekts und damit das erfolgreiche Anfahren der vorgegebenen Sollposition erkennt. 

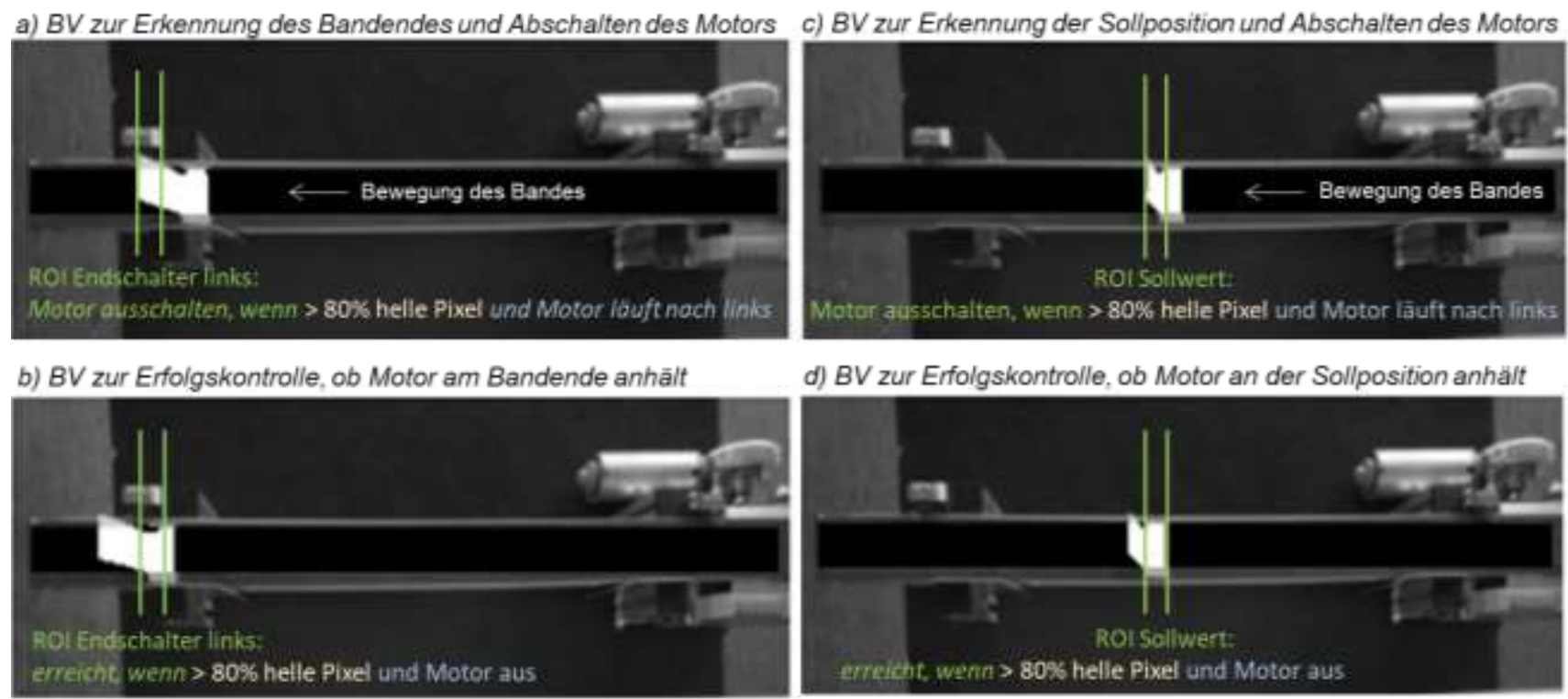

d) BV zur Erfolgskontrolle, ob Motor an der Sollposition anhallt

Bild 4: Überwachung der Position der beiden Endschalter sowie der Sollposition, die bei einer Positionsregelung vorgegeben wird

\subsection{Erkennung von Störfällen durch Bildverarbeitung}

Wenn Anwender aus der Ferne ihre Software in die Web-SPS spielen und ausprobieren, kann es passieren, dass Programmierfehler nicht gefunden und korrigiert werden und eine fehlerhafte Software in Betrieb bleibt. Oft werden beispielsweise z.B. die Endschalter verwechselt und die falsche Drehrichtung verriegelt. In der Webvisualisierung würde dann das vom Förderband bewegte Objekt über die Endschalter hinausfahren, was von der Bildverarbeitung erkannt und alarmiert wird.

Dazu ermittelt die Bildverarbeitung die 3D-Position des Objekts auf dem Band, indem Bildverarbeitungskette nach Bild 5 ausgeführt wird. Dabei werden die von der Kamera des Raspberry-Pi aufgenommenen Bilder in einer RAM-Disk gespeichert und mit dem Funktionsbaustein fb_ReadImage eingelesen und gefiltert. Dann werden die aufgenommenen Bilder innerhalb der Region of Interest (ROI) des Förderbandes binär ausgewertet. Mit Hilfe von geometrischen Merkmalen wird die Position der hellen Objekte auf dem dunklen Förderband ermittelt und nach Kalibrierung der Kamera dreidimensional skaliert.

In der HMI sind mehrere Parameter zur Konfigurierung der Bildverarbeitung einstellbar, durch die Studierende die Funktionalität der Bildverarbeitungsstufen erproben und verstehen können.

Wenn also die schnelle Kontrolle nach Bild 4 nicht erfolgreich war, erkennt die Bildverarbeitung durch den etwas langsameren Algorithmus nach Bild 5, dass das Objekt eine Position jenseits der Endschalter angefahren hat und kann dementsprechend das Förderband abschalten und eine Fehlermeldung in der HMI veranlassen.

Oft ist eine Positionsregelung notwendig, damit das Förderband z.B. eine Palette mit Werkstücken zu einer von einem Roboter vorgegebenen Position fährt und dieser die Werkstücke greifen und die Palette leerräumen kann. Hierzu kann die Positionsmessung der Bildverarbeitung genutzt und ein bildgestützter Regelkreis aufgebaut werden. Um die Totzeit infolge der zeitlichen Verzögerung der Bildauswertung zu kompensieren, wird mit Hilfe der bekannten Bandgeschwindigkeit die Position prädiziert.

Zusätzlich zu der in Bild 4d veranschaulichten Erfolgskontrolle wird durch Analyse der von der Bildverarbeitung gemessenen Position erkannt, wenn der Sollwert überschritten wird und z.B. durch schlechte Reglerparametrierung oder Störeinflüsse Überschwingungen auftreten. 


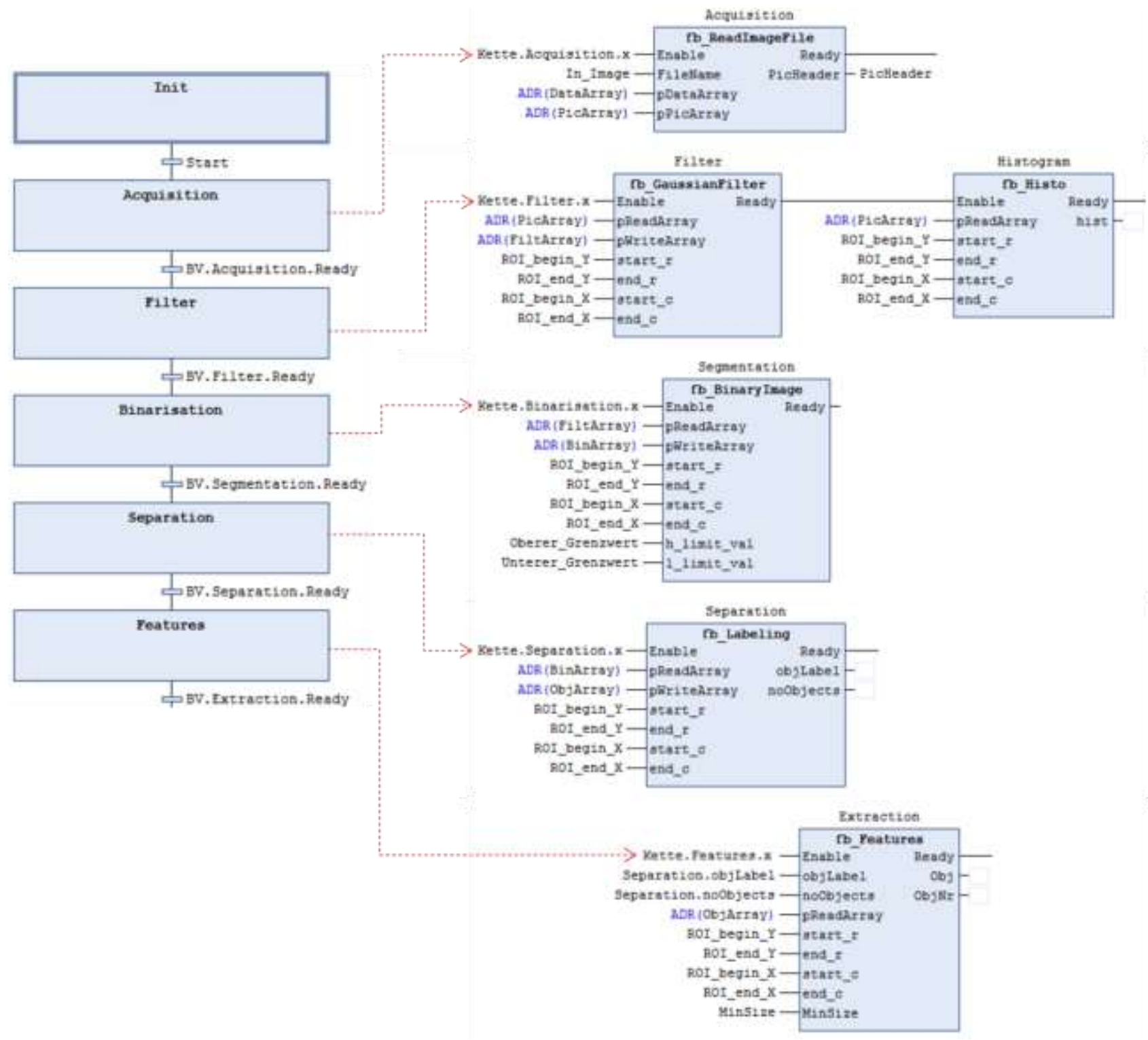

Bild 5: Bildauswertung durch Filterung, Segmentierung und Extraktion von Positionsmerkmalen

\section{Experimentelle Ergebnisse}

Früher wurde oft auf BV aus Kostengründen verzichtet. Stattdessen wurden einfachere Sensoren wie z.B. Ultraschall-Sensoren eingesetzt. Heute sind Kameras teilweise sogar günstiger als andere Sensoren. Die hier eingesetzten Kameras kosten um die 10 Euro, der Raspberry-Pi3 zur Auswertung der Bilder etwa 50 Eur. Doch Genauigkeit und Rechenaufwand sprechen oft gegen den Einsatz von Bildverarbeitung.

Deshalb wurde der Einfluss von Rechenzeitaufwand und Genauigkeit auf die bildgestützte Regelung analysiert. Die Rechenzeit für die Bildverarbeitung zur Positionsbestimmung nach Bild 5 bzw. 6b beträgt im Mittel 70 ms pro Bearbeitungszyklus. Dabei wurde mit Hilfe von SPS-Timer-Bausteinen die Zeit vom Start der Schrittkette in Bild 5 bis zum Ende der Merkmalsbestimmung gemessen. Die Bildverarbeitung zur Erfolgskontrolle quasi als Lichtschranke wie in Bild 4 dauert demnach im Mittel 20 ms pro Zyklus. Die Zykluszeit der Task im Raspberry-Pi wurde dabei auf 1 ms eingestellt.

Zusätzliche Rechenzeit beansprucht das Speichern und Anzeigen der Bilder in der Visualisierung, was aber der Anschaulichkeit der Bildverarbeitungsalgorithmen dient (s. Bild 6a). Da die Grenzwerte für 
die Binärbilderzeugung sehr empfindlich gegenüber Helligkeitsschwankungen sind, wurde eine LEDBeleuchtung angebracht, so dass auch im Dunklen die Inbetriebnahme möglich ist.

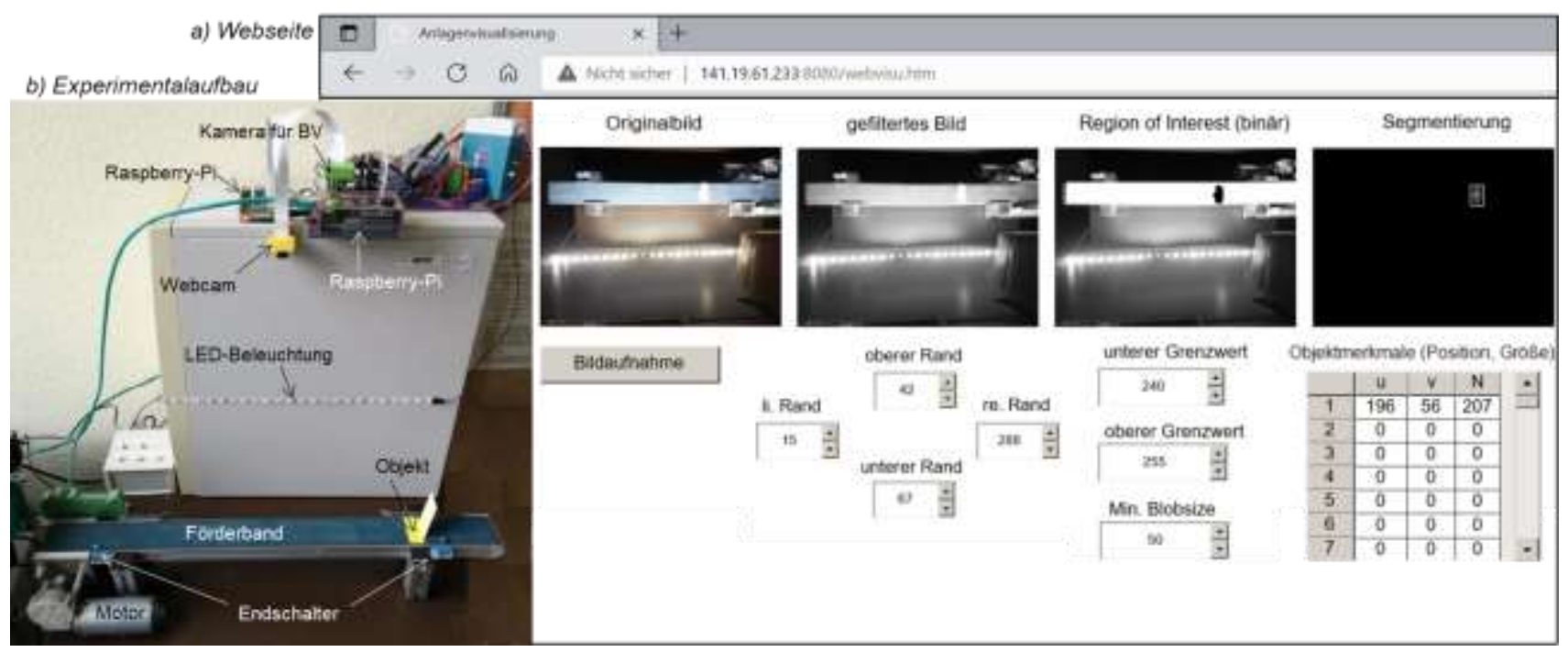

Bild 6: Experimentiersystem zur Bildüberwachung einer Förderbandanlage über das Internet

Detaillierte Aussagen zur Rechenzeit können mit dem Codesys Profiler getroffen werden, der Bestandteil des Professional Developer Pakets ist, aber 30 Tage kostenfrei getestet werden kann. Dabei zeigt sich, dass die Bildverarbeitungsbausteine in Bild 5 auch bei höherer Pixelzahl weniger als 1 ms beanspruchen. Problematisch kann jedoch die Bildaufnahme und das Einlesen der Bilder in Codesys sein, das in einzelnen Fällen max. 800 ms beansprucht hat. Vermutlich wird die Kamera dann gerade hinsichtlich Farbanpassung, Weißabgleich etc. intern kalibriert, was natürlich nicht in jedem Zyklus erforderlich ist.

Die Auswirkungen dieser Totzeiten auf die bildgestützte Positionsregelung des Objekts auf dem Band wurden durch Prädiktion der Objektposition anhand der bekannten Bandgeschwindigkeit kompensiert. Außerdem besteht noch Potenzial durch eine verbesserte Programmierung der Algorithmen, die Rechenzeiten noch weiter reduzieren zu können.

Auch die Genauigkeit der Positionsmessung durch Bildverarbeitung beeinflusst die Positionsregelung. Aufgrund der Parallaxe wird das Objekt am Rand des Gesichtsfeldes (s. Bild 4a+b) größer dargestellt als zentral unter der Kamera wie in Bild $4 c+d$. Zur Korrektur wurde die Objektfahne (s. Bild 6b), die zur Reflexion der Ultraschallwellen erforderlich ist, im Bild abgeschnitten. Weitere Ungenauigkeiten werden durch Auflösung und Linsenverzerrungen verursacht.

Tabelle 1 zeigt aber, dass die Abweichungen zwischen der Positionsmessung mit Bildverarbeitung und der Referenzmessung durch den Ultraschallsensor akzeptabel sind. Da die Objektfahne je nach Position unterschiedlich groß im Bild dargestellt wird, könnte eine positionsabhängige Korrektur der Parallaxe die Genauigkeit, z.B. am Endschalter links, noch weiter verbessern.

Tabelle 1: Genauigkeit der bildgestützten Regelung im Vergleich zur Ultraschallabstandsmessung

\begin{tabular}{|l|c|c|}
\hline Sollposition & Istposition durch BV ermittelt & Istposition durch Ultraschallsensor \\
\hline $\mathrm{x}=200 \mathrm{~mm}$ (zentral unter Kamera) & $197,2 \mathrm{~mm}$ & $200,1 \mathrm{~mm}$ \\
\hline $\mathrm{x}=35 \mathrm{~mm}$ (Endschalter links) & $31 \mathrm{~mm}$ & $35,2 \mathrm{~mm}$ \\
\hline $\mathrm{x}=470 \mathrm{~mm}$ (Endschalter rechts) & $471 \mathrm{~mm}$ & $470 \mathrm{~mm}$ \\
\hline
\end{tabular}




\section{Schlussfolgerungen}

Es wurde gezeigt, dass ein Remote-Engineering über das Internet sehr einfach über eine VPN-Verbindung der SPSen mit dem Programmiersystems möglich ist. Das Cloud-basierte Engineering mit dem Codesys Automation Server ist nur mit Codesys-Steuerungen möglich. SPS-Hersteller wie ABB haben ihre eigenen Cloudsysteme, die nicht ohne Weiters mit dem Automation Server kompatibel sind.

Zur automatisierten Erfolgskontrolle und Fehlererkennung wurden zwei Bildverarbeitungsmethoden eingesetzt. Zunächst erkennt die Bildverarbeitung in der Region of Interest die erreichte Position des Objekts, so dass der Förderbandantrieb automatisch angehalten werden kann. Wenn dies in Folge von Programmierfehlern oder Störungen nicht erfolgt, erkennt dies die Bildverarbeitung durch Messung der unerlaubten Objektposition. Außerdem wurde mit Hilfe der Bildverarbeitung die Positionsregelung des Objekts auf dem Förderband entwickelt. Dabei wird die Geschwindigkeit des Bandes reduziert je näher das Objekt an die Sollposition gelangt.

Studierende mit VPN-Zugang können somit an dieser Experimentieranlage programmieren und jederzeit ihre Software in die SPS laden und ausprobieren. Durch Kameras werden die Auswirkungen der entwickelten Programme beobachtet und analysiert. Diese Remote-Inbetriebnahme ermöglicht es, die Software so lange zu korrigieren, bis das gewünschte Verhalten erreicht wird.

\section{Literatur}

[1] Baker Jr., R. A. (2000). The Web PLC is here. In: Machine Design, https://www.machinedesign.com/ mechanicalmotion-systems/controllers/article/21832452/the-web-plc-is-here

[2] Langmann, R. (2005). Web-based Remote Control by LiveConnect. International Journal of Online Engineering.

[3] Mühlbauer, A. (2019). Die HMI geht ans Netz. In: Industrial Production

[4] Codesys (2021). Automation Server - Die Industrie-4.0-Plattform. https://www.automation-server.com/de

[5] Goldstein, S. (2021): Engineering leicht gemacht - IoT-Konzepte für die Automatisierung mit TwinCAT Cloud Engineering, SPS-Magazin 11/2021

[6] Siemens (2021). Simatic IoT2050, https://new.siemens.com/de/de/produkte/automatisierung/pc-based/iot-gateways/simatic-iot2050.html

[7] Seitz, M. (2021) Speicherprogrammierbare Steuerungen in der Industrie 4.0, 5. Auflage, Hanser Verlag, München.

[8] Papenfort, J. (2017). Bildverarbeitung in der SPS TwinCAT Vision - Einfache Integration der Bildverarbeitung in die Automatisierungstechnik. PC-Control 04/2017

[9] B\&R (2019). Integrierte Bildverarbeitung sichert Prozess- und Produktqualität. In: Automation 11.19

[10] Seitz, M. (2021). SPS-Lern-und-Übungsseite, www.seitz.et.hs-mannheim.de

[11] IEC 62433-4 (2018). Security for industrial automation and control systems, VDE-Verlag.

[12] Gottschalt, D., Peter, H., Seitz, M. (2016). SPS-integrierte Bildverarbeitung. In: A\&D-Kompendium 2016/2017. 